\title{
The Prognostic Significance of Hsp70/Hsp90 Expression in Breast Cancer: A Systematic Review and Meta-analysis
}

\author{
DIONYSIOS TH. DIMAS ${ }^{1}$, CHRISTINA D. PERLEPE ${ }^{1,2}$, THEODOROS N. SERGENTANIS ${ }^{2}$, \\ IOANNIS MISITZIS ${ }^{3}$, KONSTANTINOS KONTZOGLOU ${ }^{4}$, EFSTRATIOS PATSOURIS ${ }^{5}$, \\ GREGORY KOURAKLIS ${ }^{4}$, THEODORA PSALTOPOULOU ${ }^{2}$ and AFRODITI NONNI ${ }^{5}$ \\ ${ }^{1}$ Department of Surgery, "Elpis" General Hospital of Athens, Athens, Greece; \\ ${ }^{2}$ Department of Hygiene, Epidemiology and Medical Statistics, \\ Medical School, National University of Athens, Athens, Greece; \\ ${ }^{3}$ Breast Cancer Surgical Department, "Ag. Savvas" Anticancer Hospital, Athens, Greece; \\ ${ }^{4}$ Second Department of Propedeutic Surgery, Medical School, \\ University of Athens, "Laikon" General Hospital, Athens, Greece; \\ ${ }^{5}$ First Department of Pathology, Medical School, National and Kapodistrian University of Athens, Athens, Greece
}

\begin{abstract}
Background/Aim: Studies have focused on heat shock protein (Hsp) inhibitors as potential treatment agents in breast cancer, with controversial results. Adopting a pathophysiological perspective, this systematic review aims to synthesize the evidence examining the association between Hsp70/Hsp90 expression and breast cancer prognosis, as well as prognosis-related clinicopathological indices. Secondarily, changes in Hsp70/Hsp90 expression in the continuum of breast neoplasia were assessed. Materials and Methods: Hsp70/ Hsp90 expression was approached globally, quantified by means of immunohistochemistry, western blot or PCR. This study was performed in accordance with the PRISMA guidelines. Relevant studies were sought in PubMed, up to December 31,2015. Results: A total of 23 eligible studies were identified ( 7,288 breast cancer cases). High Hsp 90 expression was associated with worse overall survival (pooled $R R=1.48$, 95\%CI=1.21-1.82) and marginally with worse disease-free survival. High Hsp70 expression also correlated with worse disease-free survival (pooled $R R=1.77,95 \% C I=1.71-2.82$ ). $H$ sp70 intense expression correlated with ER positivity (pooled $O R=3.51,95 \% C I=1.31-9.40$ ) and $P R$ positivity (pooled $O R=2.48,95 \% C I=1.39-4.44)$. No significant associations were noted between Hsp70/Hsp90 expression and clinicopathological variables including histological grade, tumor size,
\end{abstract}

Correspondence to: Theodoros N. Sergentanis, Department of Hygiene, Epidemiology and Medical Statistics, Medical School, National and Kapodistrian University of Athens, 75 M. Asias Str., Athens 115 27, Greece. Tel: +30 2107462061, e-mail: tsergentanis@yahoo.gr

Key Words: Hsp90, Hsp70, meta-analysis, systematic review, survival, estrogen receptor. nodal metastasis or patient age at diagnosis. No clear pattern emerged for Hsp70/Hsp90 expression along the breast neoplasia continuum. Conclusion: This systematic review and meta-analysis highlights the prognostic role of $\mathrm{Hsp} 90$ and Hsp70 expression in breast cancer. Further high-quality studies, with detailed reporting are needed to provide epidemiological evidence complementing the findings of ongoing clinical trials on Hsp inhibitors.

Heat shock proteins are well maintained molecules, that are overexpressed in cells subjected to various stress stimuli (such as heat shock or disruption of homeostasis). They act as molecular chaperones assisting the folding of proteins in normal metabolic conditions and amplifying the levels of repair and stabilization of proteins in the face of molecular stress (1). It has been suggested that they play an important part in cellular defense against carcinogenesis (2). Their role in tumorigenesis includes the stabilization of oncogenic proteins, inhibition of programmed cell death, inhibition of replicative senescence, induction of tumor angiogenesis and possibly the activation of invasion and metastasis (1). Interestingly, the majority of the published studies on Hsp70 and Hsp90 expression stems from breast cancer cell lines and xenografts and not from actual biopsy samples $(3,4)$.

Various anti-Hsp agents have been evaluated as complements to the standard breast cancer treatment regimens. From the completed phase 1 clinical trials on Hsp90 inhibitors a low clinical activity, at least in an orally administered form $(5,6)$, but various toxicities $(6)$, such as night blindness $(4,7)$, have been noted. A phase 2 clinical trial on the use of Hsp90 inhibitor ganetespib showed tolerable toxicity levels, and suggested a possible beneficial effect in selected patient subgroups (8). 
Numerous studies have appeared in the literature examining the role of Hsp70/Hsp90 protein/mRNA expression in breast carcinogenesis, undertaking a variety of research designs, analyses and quantification methods (immunohistochemistry, Western blot and PCR). This systematic review aims to synthesize the results from studies assessing: i. the prognostic role of Hsp70/Hsp90 expression (effects upon overall/diseasefree survival), ii. their associations with meaningful prognosis-related biomarkers and clinicopathological characteristics, including age, histological grade, tumor size, nodal status, ER, PgR, HER2/neu, Ki-67 and PCNALI and iii. the changes in Hsp70/Hsp90 expression along the continuum of normal breast tissue, precursor, pre-invasive and invasive breast lesions. Studies evaluating the effects of interventions with Hsp70 and Hsp90 inhibitors were not examined, as the scope of the present systematic review pertains to the physiological associations.

\section{Materials and Methods}

Search strategy and eligibility of studies. This systematic review and meta-analysis was performed according to the PRISMA guidelines and followed the a priori protocol agreed upon and signed by all authors. Eligible articles were identified through a search of PubMed database for the period up to December 31, 2015. The search algorithm used is the following: (hsp OR (heat shock protein) OR hsp70 OR hsp90) AND breast AND (cancer OR carcinoma* OR malignan* OR neoplasm* OR tumour OR tumor OR sarcoma* OR leiomyosarcoma*). Reference lists of relevant articles were scrutinized for potentially eligible studies ("snowball" procedure), in order to identify additional potentially relevant articles.

Studies referring to cancer cell lines, xenografts, in vitro and animal studies, reviews, and cases where chemotherapy, hormonal or irradiation therapy was performed prior to Hsp70/Hsp90 evaluation, studies comparing the expression of Hsps in metastases versus the primary tumor were excluded from the present review. In addition to the systematic review of the literature, an additional unpublished cohort $(n=54)$ supplied by the first author was included in the present systematic review (mentioned as "Dimas, unpub." throughout the text) (9). The details about patients and methods regarding this study are presented in the Supplemental File A (https://s3.eu-central1.amazonaws.com/dimas-et-al/Supplemental+A.docx).

Two authors (DD and CP) working independently and blindly to each other performed the selection of eligible studies. In case of a disagreement a consensus with the whole team was reached.

Data extraction and effect estimates. Relevant details are presented in Supplemental File B (https://s3.eu-central-1.amazonaws.com/ dimas-et-al/Supplemental+B.docx).

Statistical analysis and risk of bias. Random-effects (DerSimonianLaird) models were used to calculate pooled effect estimates, as appropriate. Between-study heterogeneity was assessed through Cochran Q statistic and by estimating $\mathrm{I}^{2}(10)$.

Regarding the associations between Hsp70/Hsp90 expression and (overall or relapse free) survival, pooling of hazard ratios / relative risks was performed, given the underlying cohort design. On the other hand, concerning the associations between clinicopathological

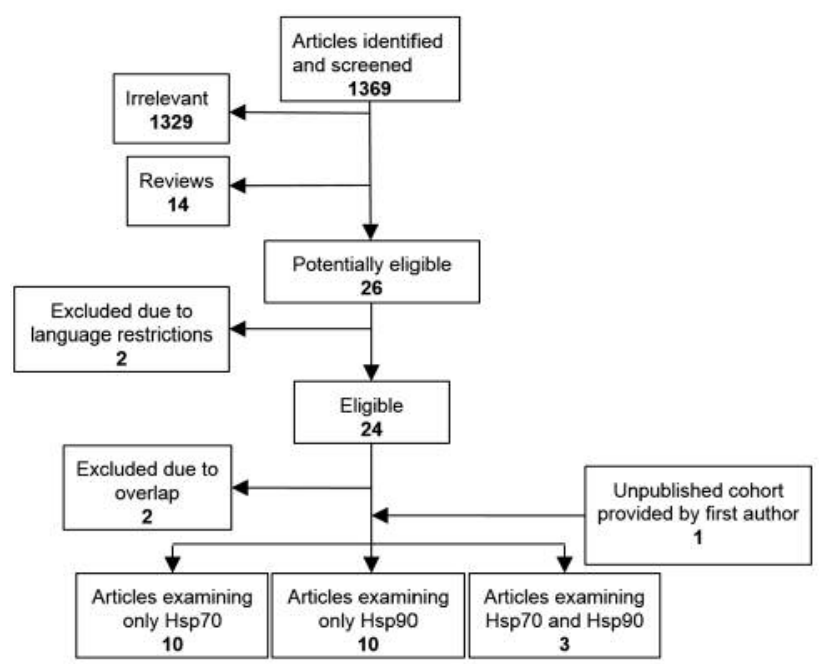

Figure 1. Flow chart presenting the successive steps during the selection of eligible studies.

variables and Hsp70/Hsp90 expression, the relevant odds ratios were pooled. A comparison between "highest" vs. "lowest" expression categories was adopted, as in our previous meta-analyses $(11,12)$.

Meta-analysis was performed only for outcomes examined by three or more study arms. Nevertheless, given that the main outcome of this meta-analysis was survival, an explorative synthesis based on two studies was undertaken for (overall or relapse free) survival. Statistical analysis was performed using STATA/SE version 13 (Stata Corp, College Station, TX, USA).

Regarding the risk of bias, the quality of the included studies was evaluated using the Newcastle-Ottawa Quality scale, separately for cohort, cross-sectional and case-control studies (13). No assessment of publication bias was undertaken, given that the number of synthesized studies was considerably less than 10 (10-12).

\section{Results}

Selection and description of eligible studies. The aforementioned algorithm rendered 1369 results, of which 23 studies were deemed eligible. Details about the selection of studies are presented in Supplemental File B (https://s3.eucentral-1.amazonaws.com/dimas-et-al/Supplemental+B.docx ) (2, 9, 14-34). Figure 1 presents the PRISMA flow chart for the selection of studies.

Table I presents the characteristics of the included studies. Table II illustrates the results pertaining to the association between Hsp70/Hsp90 and the development of metastases, and survival. Finally, Table III summarizes the correlations between the expression of Hsp70/Hsp90 and the various clinicopathological characteristics examined in each study.

Hsp90 expression, subsequent development of metastases and survival. Three studies examined the impact of Hsp90 
Table I. Characteristics of the included studies.

\begin{tabular}{lccccccc}
\hline $\begin{array}{l}\text { Study } \\
\text { publication }\end{array}$ & Country & $\begin{array}{c}\text { Sample } \\
\text { size }\end{array}$ & Age & $\begin{array}{c}\text { Follow-up } \\
\text { range }\end{array}$ & $\begin{array}{c}\text { Hsp70 } \\
\text { mean or } \\
\text { assessment }\end{array}$ & $\begin{array}{c}\text { Hsp90 } \\
\text { assessment }\end{array}$ & $\begin{array}{c}\text { Type of } \\
\text { breast } \\
\text { lesions }\end{array}$
\end{tabular}

Tauchi et al., Japan $59(+5 \quad$ N/R $\quad$ N/A $\quad$ yes no $\quad 59$ breast $\quad$ Mouse monoclonal antibody against

1991 (27) benign) carcinomas,

5 fibroadenomas

$\begin{array}{lcccccr}\begin{array}{l}\text { Ciocca } \text { et al., } \\ 1993(16)\end{array} & \text { USA } & 507 & \text { N/R } & 8 \text { years } & \text { yes } & \text { no } \\ \begin{array}{l}\text { Jameel } \text { et al., } \\ 1993(18)\end{array} & \text { UK } & \begin{array}{c}123(+17 \\ \text { normal })\end{array} & 25-88 & 7 \text { years } & \text { no } & \text { yes }\end{array}$

Shyamala et al., Canada 1993 (22)

Takahashi et al., Japan 1994 (25)

23

121

(1)

$\mathrm{N} / \mathrm{R}$

yes

no

es

(1)
negative breast carcinoma

Invasive breast carcinoma, IDC (76.42\%),

ILC $(8.13 \%)$, papillary $(1.63 \%)$, others $(11.38 \%)$

Breast neoplasias

Invasive human breast carcinoma,
Takahashi et al., Japan 1995 (26)
Yano et al., Japan 131 1996 (31)

\begin{tabular}{|c|c|c|c|c|c|}
\hline $\begin{array}{l}\text { Franzen et al., } \\
1997 \text { (17) }\end{array}$ & Sweden & 23 & $\mathrm{~N} / \mathrm{R}$ & N/A & no \\
\hline $\begin{array}{l}\text { Lazaris et al., } \\
1997 \text { (20) }\end{array}$ & Greece & 80 & $29-65$ & N/A & yes \\
\hline $\begin{array}{l}\text { Vargas-Roig } \\
\text { et al., } 1997 \text { (30) }\end{array}$ & Argentina & 40 & $22-70$ & N/A & yes \\
\hline
\end{tabular}
et al., 1997 (30) medullary $(1.63 \%)$, colloid $(0.81 \%)$ and IDC $(88.71 \%)$,

ILC (1.61\%),

Mixed (2.42\%),

Medullary carcinoma

(3.23\%), Mucinous

$(2.42 \%)$, Apocrine

Carcinoma (1.61\%)

Invasive human

breast carcinoma, IDC (88.71\%), ILC (1.61\%), Mixed (2.42\%),

Medullary carcinoma

(3.23\%), Mucinous

(2.42\%), Apocrine

Carcinoma (1.61\%)

Papillotubular carcinoma (35.88\%), solid tubular carcinoma (14.50\%), scirrhous carcinoma $(37.40 \%)$, other types $(12.21 \%)$

Hsp70 (RPN-1197, Amersham), mouse monoclonal antibody against c-myc (OM-11-906, Cambridge Research

Biochemicals), Western blot

Mouse monoclonal antibody C92 for Hsp70, Western blot

PCR and Northern blotting

\section{Western blot}

Mouse monoclonal antibody to EGF-R1

(Amersham Japan, Tokyo, Japan),

anti-p53 antibodies, clones PAb 1801,

PAb 1620 and PAb 240 (Oncogene

Science, Manhasset, NY), anti-c-erbB-2 antibody, clone NCL-CB11

(Novocastra Laboratories, Newcastle, UK). Anti-Hsp70, clone C92 (Amersham)

Mouse anti-ER monoclonal antibody, clone 1D5 (Dako Japan, Kyoto, Japan)

Mouse monoclonal antibody BRM-22 against the constitutive and inducible forms of Hsp70

(Sigma), mouse monoclonal antibody PC10 against PCNA (Novocastra Laboratories, UK) and silver staining nucleolar organizer regions(AgNORs). mRNA in situ hybridization (ISH)

4 fibroadenomas,

2 other benign

breast lesions, 17 malignant carcinomas

Primary ductal infiltrative carcinoma not otherwise specified IDC $(85 \%)$, Immunoblotting

Dako monoclonal antibody to PCNA (clone PC-10), Dako polyclonal antibody to $\mathrm{Hsp} 70$ Mouse monoclonal antibody BRM-22 Medullary Carcinoma against the constitutive and inducible
$5 \%$ ), Colloid Carcinoma forms of Hsp70(Sigma), mouse $(2.5 \%)$, Papillary monoclonal antibody PC10 against Carcinoma (2.5\%), PCNA (Novocastra Laboratories, UK) ILC $(2.5 \%)$, other $(2.5 \%)$ and silver staining nucleolar organizer regions (AgNORs), Western blot 
Table I. Continued

\begin{tabular}{|c|c|c|c|c|c|c|c|c|}
\hline $\begin{array}{l}\text { Study } \\
\text { publication }\end{array}$ & Country & $\begin{array}{l}\text { Sample } \\
\text { size }\end{array}$ & $\begin{array}{l}\text { Age } \\
\text { range }\end{array}$ & $\begin{array}{l}\text { Follow-up } \\
\text { (mean or } \\
\text { median) }\end{array}$ & $\begin{array}{l}\text { Hsp70 } \\
\text { assessment }\end{array}$ & $\begin{array}{c}\text { Hsp90 } \\
\text { assessment }\end{array}$ & $\begin{array}{l}\text { Type of } \\
\text { breast } \\
\text { lesions }\end{array}$ & $\begin{array}{l}\text { Method } \\
\text { of Hsp } \\
\text { assessment }\end{array}$ \\
\hline $\begin{array}{l}\text { Thanner et al., } \\
2003 \text { (28) }\end{array}$ & Germany & 191 & $24-86$ & 14.75 years & yes & no & $\begin{array}{c}\text { Invasive ductal } \\
\text { carcinoma }(80.23 \%), \\
\text { other node negative } \\
\text { breast carcinoma } \\
\quad(19.77 \%)\end{array}$ & $\begin{array}{c}\text { Monoclonal anti-Hsp70 } \\
\text { (Biogenix, San Ramon, USA) }\end{array}$ \\
\hline $\begin{array}{l}\text { Torronteguy } \\
\text { et al., } 2006 \text { (29) }\end{array}$ & Brazil & 53 & $\mathrm{~N} / \mathrm{R}$ & 3 years & yes & no & $\begin{array}{c}\text { IDC }(64.15 \%), \\
\text { ILC }(18.86 \%), \\
\text { Mucinous Carcinoma } \\
(3.7 \%), \text { Metaplastic } \\
\text { Carcinoma }(1.88 \%), \\
\text { DCIS }(3.77 \%)\end{array}$ & $\begin{array}{c}\text { Antibodies against Hsp70 (Stressgen) } \\
\text { and IFN-g (R\&D Systems, } \\
\text { Minneapolis, MN) for } \\
\text { immunohistochemistry. } \\
\text { Anti-human Hsp70 antibody } \\
\text { (SPA810, Stressgen) or anti- } \\
\text { HSP/Hsc70 (SPA820, Stressgen) } \\
\text { for Western blot analysis. }\end{array}$ \\
\hline $\begin{array}{l}\text { Kalogeraki et al., } \\
2007 \text { (19) }\end{array}$ & ., Greece & 50 & $\mathrm{~N} / \mathrm{R}$ & N/A & yes & no & IDC & $\begin{array}{l}\text { Monoclonal antibodies to Hsp70 } \\
\text { Ad-2(clone w27), MIB1 and ER } \\
\text { and a standard alcaline phosphatase } \\
\text { APAAP method. }\end{array}$ \\
\hline
\end{tabular}

Pick et al., $\quad$ USA $655 \quad$ N/R 10 years no $\quad$ yes 331 node negative and 324 node positive 2007 (21) breast cancer cases

Mouse monoclonal anti-HSP90, mouse monoclonal anti-ER (Dako,

Carpinteria, CA), mouse monoclonal anti-PR (Dako, Carpinteria, CA), rabbit anti-Her2/neu (Dako,

Carpinteria, CA). Mouse anti-HSP90 IgG (BD Transduction, San Jose, CA) for Western blot analysis Rabbit polyclonal anti-vimentin antibody (Boster Biological Technology), mouse polyclonal anti-actin antibody (Zhongshan), mouse polyclonal anti-HSP70 antibody (Zhongshan), rabbit polyclonal anti-HSP90a antibody (Newmarker), rabbit polyclonal anti-CK18 antibody (Maxim,

Biotecho), rabbit polyclonal anti14-3-3h antibody (Newmarker), rabbit polyclonal anti-tubulin-h antibody (Newmarker), and mouse monoclonal anti-moesin antibody (Newmarker)

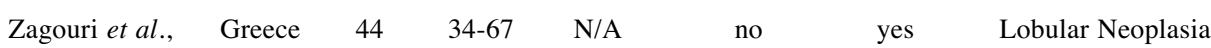
2008 (32) of the Breast

Diehl et al.

DCIS $(9.33 \%)$, IDC $(72.66 \%)$, Normal tissue sample as controls $(18 \%)$ Batchelder et al., UK $120 \quad$ N/R N/A yes no 58 breast cancer, 2009 (14)

$120 \quad \mathrm{~N} / \mathrm{R} \quad \mathrm{N} / \mathrm{A} \quad$ yes no
62 served as controls

Monoclonal antibody Hsp90 (clone JPB24, Novocastra, supplied by Menarini), antibodies ER-alpha (ER6f-11) and ER-beta (clone EMRO2,

Novocastra, supplied by Menarini) Antibodies against Hsp90 and antibodies against $\mathrm{p} 23$ by use of

VectaStain $\mathrm{ABC}$ immunoperoxidase IgG detection kit (Vector Labs, Burlingame, CA), Western blot

Hsp70 mouse monoclonal antibody (NCL-Hsp70, Novocastra Limited, UK), Ki-67 mouse monoclonal antibody (NCL-Ki67-MM2,

Novocastra Limited, UK) and others unrelated to the current study 
Table I. Continued

\begin{tabular}{|c|c|c|c|c|c|c|c|c|}
\hline $\begin{array}{l}\text { Study } \\
\text { publication }\end{array}$ & Country & $\begin{array}{l}\text { Sample } \\
\text { size }\end{array}$ & $\begin{array}{l}\text { Age } \\
\text { range }\end{array}$ & $\begin{array}{l}\text { Follow-up } \\
\text { (mean or } \\
\text { median) }\end{array}$ & $\begin{array}{c}\text { Hsp70 } \\
\text { assessment }\end{array}$ & $\begin{array}{c}\text { Hsp90 } \\
\text { assessment }\end{array}$ & $\begin{array}{l}\text { Type of } \\
\text { breast } \\
\text { lesions }\end{array}$ & $\begin{array}{l}\text { Method } \\
\text { of Hsp } \\
\text { assessment }\end{array}$ \\
\hline $\begin{array}{l}\text { Zagouri et al., } \\
2010 \text { (34) }\end{array}$ & Greece & 163 & $31-78$ & N/A & no & ( & $\begin{array}{c}\text { Ductal Hyperplasia } \\
\text { (DH) without Atypia } \\
(18.40 \%) \text {, Atypical DH } \\
(19.02 \%) \text {, DCIS }(31.29 \%), \\
\text { IDC }(31.29 \%)\end{array}$ & $\begin{array}{c}\text { Mouse monoclonal antibody Hsp90 } \\
\text { (clone JPB24, NCL-Hsp90, } \\
\text { Novocastra, supplied by Menarini), } \\
\text { antibody PR (636,Dako), } \\
\text { antibody ER (ID5, Dako), antibody } \\
\text { c-erbB-2 (CB11, Novocastra) }\end{array}$ \\
\hline $\begin{array}{l}\text { Zagouri et al., } \\
2010 \text { (33) }\end{array}$ & Greece & 32 & $35-74$ & N/A & no & yes & ILC & $\begin{array}{c}\text { Mouse monoclonal antibody } \\
\text { Hsp90 (clone JPB24, NCL-Hsp90, } \\
\text { Novocastra, supplied by Menarini), } \\
\text { antibody PR (636, Dako), antibody } \\
\text { ER (ID5, Dako), antibody c-erbB-2 } \\
\text { (CB11, Novocastra) }\end{array}$ \\
\hline $\begin{array}{l}\text { Song et al., } \\
2010 \text { (23) }\end{array}$ & Korea & 212 & $\mathrm{~N} / \mathrm{R}$ & 5 years & no & yes & $\begin{array}{c}\text { IDC }(96.7 \%) \text {, } \\
\text { ILC }(3.3 \%) \\
\text { Subtypes were } \\
\text { defined as follows: } \\
62 \% \text { luminal A, } \\
9 \% \text { luminal B, } \\
13 \% \text { HER2+/ER-, } \\
18 \% \text { triple-negative }\end{array}$ & $\begin{array}{l}\text { Dako Envision detection kit (Dako, } \\
\text { Carpinteria, CA), specifically: Hsp90 } \\
\text { (Cell Signaling Technology), ER (SP1, } \\
\text { Labvision), PR(PgR636), HER-2, } \\
\text { Ki-67 (Dako), and others unrelated } \\
\text { to the current study }\end{array}$ \\
\hline $\begin{array}{l}\text { Cheng et al., } \\
2012 \text { (15) }\end{array}$ & $\begin{array}{l}\text { USA, } \\
\text { Germany, } \\
\text { Belgium, } \\
\text { France, } \\
\text { Sweden, } \\
\text { Singapore, } \\
\text { Netherlands }\end{array}$ & 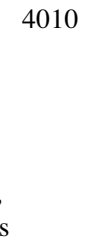 & $\mathrm{N} / \mathrm{R}$ & $\mathrm{N} / \mathrm{R}$ & no & yes & $\begin{array}{c}\text { HER2 }(+)(25.23 \%) \\
\text { HER2 }(-) / \text { ER }(+) \\
(48.86 \%), \text { TNBC } \\
(25.91 \%)\end{array}$ & Depends on the respective dataset \\
\hline $\begin{array}{l}\text { Dimas (un- } \\
\text { published } \\
\text { dataset } \\
\text { provided by } \\
\text { first author) }\end{array}$ & Greece & 54 & $42-81$ & N/A & yes & yes & IDC & $\begin{array}{l}\text { Monoclonal Hsp70 antibody (NCL- } \\
\text { Hsp70, clone } 8 \text { B } 11 \text {, Novocastra, } \\
\text { supplied by Menarini), monoclonal } \\
\text { Hsp90 antibody (NCL-Hsp90, clone } \\
\text { JPB24, Novocastra, } \\
\text { supplied by Menarini) }\end{array}$ \\
\hline
\end{tabular}

a: The two studies by Takahashi et al. examine the same cohort of patients; ILC: infiltrative lobular carcinomas, DCIS: ductal carcinoma in situ, N/R: not reported, N/A: not applicable.

expression on overall survival (OS); one adopted a continuous score $(21)$, whereas the remaining two $(15,18)$ adopted a comparison between high and low Hsp90 expression categories. The synthesis of the latter yielded a significant association between poorer OS and high Hsp90 expression (pooled $\mathrm{RR}=1.48,95 \% \mathrm{CI}=1.21-1.82$ ), (Figure $2 a)$. Two studies proceeded to a further analysis by cancer subtype. Cheng et al. showed no association in HER2(+) and TNBC subgroups separately, but a significant association with worse OS in the HER2(-)/ER(+) subgroup (15). Pick et al. studied node positive and node negative patients separately and found a worse OS in node negative patients with high Hsp90 expression, but no difference in the node positive subgroup (21). DSS was only examined by Cheng et al. who showed a worse survival in patients with higher Hsp90 expression (15).

As far as disease free survival (DFS) is concerned, Song et al. examining all types of breast carcinomas showed a worse DFS in patients with high Hsp90 expression, which however was significant only in the univariate analysis (23). The same significant association was noted, regarding relapse free survival (RFS) by Jameel et al. (18). Cheng et al. concurred to this conclusion, but this result pertained only to TNBC patients and no association could be established when studying all cancer subtypes combined or the HER2(-)/ER(+) or HER2(+) subgroups (15). The exploratory synthesis of studies providing effect estimates yielded an association of borderline significance (pooled $\mathrm{RR}=1.46,95 \% \mathrm{CI}=0.98-2.16$, 
Table II. Association between Hsp70 and Hsp90 expression with survival. (+) Stands for a significant positive correlation, (-) for an inverse correlation and (0) for a null association.

\begin{tabular}{|c|c|c|c|}
\hline Study publication & $\begin{array}{l}\text { Presence of } \\
\text { metastases }\end{array}$ & $\begin{array}{c}\text { Overall } \\
\text { survival }\end{array}$ & $\begin{array}{l}\text { Disease free survival/ } \\
\text { Relapse free survival }\end{array}$ \\
\hline Ciocca et al., 1993 (16) & & (0) Hsp70 & worse in high Hsp70 \\
\hline Jameel et al., 1993 (18) & & worse in high Hsp90a & worse in high Hsp90a \\
\hline Thanner et al., 2003 (28) & & $\begin{array}{l}\text { worse in high Hsp70 cytoplasmic, (0) } \\
\text { Hsp70 nuclear }\end{array}$ & (0) Hsp70 cytoplasmic and nuclear \\
\hline Torronteguy et al., 2006 (29) & (-) Hsp70 & (0) Hsp70 & (0) Hsp70 \\
\hline Pick et al., 2007 (21) & & $\begin{array}{l}\text { worse in high Hsp90, (0) Hsp90 for } \\
\text { node positive patients' subgroup }\end{array}$ & \\
\hline Sun et al., 2008 (24) & $\begin{array}{c}\text { (+) Hsp70 in TNBC, } \\
(0) \mathrm{Hsp} 70 \mathrm{ER}(+) / \mathrm{PR}(+) \\
(0) \mathrm{Hsp} 90\end{array}$ & & \\
\hline Song et al., 2010 (23) & & & $\begin{array}{c}\text { worse in high Hsp90, ss only in } \\
\text { univariate analysis }\end{array}$ \\
\hline Cheng et al., 2012 (15) & $\begin{array}{l}\text { (0) Hsp90 for all types combined } \\
(0) \text { Hsp90 in TNBC } \\
(+) \text { Hsp90 non ss in HER2(-)/ER(+) } \\
(0) \text { Hsp90 in HER2(+) }\end{array}$ & $\begin{array}{c}\text { worse in high Hsp90 for all types } \\
\text { (0) Hsp90 in TNBC } \\
\text { worse in high Hsp90 in HER2(-)/ER(+) } \\
(0) \text { Hsp90 in HER2(+) }\end{array}$ & $\begin{array}{l}\text { worse in high Hsp90 non ss in TNBC } \\
\text { (0) Hsp90 for all types combined } \\
\text { worse in high Hsp90 in TNBC } \\
\text { (0) Hsp90 in HER2(-)/ER(+) } \\
\text { (0) Hsp90 in HER2(+) }\end{array}$ \\
\hline
\end{tabular}

ss: Statistically significant.

$p=0.06$ ). (See also Supplemental File B (https://s3.eu-central1.amazonaws.com/dimas-et-al/Supplemental+B.docx)).

No association was noted between Hsp90 expression and metastases by two studies $(15,24)$; only a weak association has been reported in the HER2(-)/ER(+) subgroup (15).

Hsp90 expression through the continuum of breast neoplasia. Yano et al. referring to all types of breast cancer (mRNA ISH, Hsp90a arm), Diehl et al. (regarding cytoplasmic, but not nuclear, expression in ductal carcinomas) and Zagouri et al., 2010 (only for the comparison between IDC $v s$. normal breast tissue, and with the exception of the TNBC subgroup) showed that there is a significantly higher $\mathrm{Hsp} 90$ expression in cancerous tissues compared to the non-cancerous ones $(2,31,34)$. Franzen et $a l$. noted an increased Hsp90 expression in highly malignant breast carcinomas in comparison to benign breast lesions, but no statistical testing was performed (17). In contrast to that, the TNBC arm of the Zagouri et al. study and the Dimas, unpub. cohort pointed to a significantly lower Hsp90 expression, in ductal carcinoma tissues compared to noncancerous ones $(9,34)$. Moreover, the Dimas, unpub. data indicated a reduction also evident between IDC and DCIS, as well as DCIS and normal breast tissue. Especially concerning lobular lesions Zagouri et al. showed a significantly lower Hsp90 expression in lobular neoplasia and invasive lobular carcinomas, compared to the noncancerous breast tissue $(32,33)$.
Hsp90 expression in conjunction with clinicopathological variables. Three studies examined Hsp90 expression in different age groups and found no association $(9,18,21)$.

No clear conclusions could be reached regarding the association between histological grade and Hsp90 expression. Yano et al. examining all types of breast carcinoma, showed a significant positive association, only concerning Hsp90b (31). Zagouri et al., examining ductal neoplasia of the breast, showed a non-significant positive association (34). Dimas, unpub., referring to ductal neoplasias of the breast, Song et al. examining all types of invasive breast carcinomas, and the Hsp90a arm by Yano et al. showed no association between Hsp90 expression and histological grade $(9,23,31)$.

Concerning tumor size two studies found a significantly positive association with high Hsp90 expression (21, 34). On the other hand, Song et al., Jameel et al. and Dimas, unpub. found no association $(9,18,23)$.

The results pertaining to nodal metastases at the time of diagnosis were not conclusive. Jameel et al. and Pick et al. referring to all breast carcinoma types, showed a nonsignificant (only in histologically confirmed nodal status) and significant, respectively, association $(18,21)$. Three more studies suggested an absence of association $(9,23,34)$.

Regarding the expression of estrogen receptors (ER), two studies showed a significantly positive correlation with Hsp90, one referring to all types of invasive breast carcinoma (21) and the other only to ductal neoplasia (this association 
Table III. Correlations between Hsp70/Hsp90 expression and the clinicopathological variables examined in each study. (+) stands for a significant positive correlation, (-) for an inverse correlation and (0) for a null association.

\begin{tabular}{|c|c|c|c|c|c|c|c|c|c|c|}
\hline $\begin{array}{l}\text { Study } \\
\text { publication }\end{array}$ & Age & $\begin{array}{l}\text { Cancer vs. } \\
\text { non cancer }\end{array}$ & $\begin{array}{l}\text { High } \\
\text { grade }\end{array}$ & $\begin{array}{l}\text { Large tumor } \\
\text { size }\end{array}$ & $\begin{array}{l}\text { Nodal } \\
\text { status }\end{array}$ & $\begin{array}{c}\text { ER } \\
\text { expression }\end{array}$ & $\begin{array}{c}\text { PR } \\
\text { expression }\end{array}$ & $\begin{array}{l}\text { HER2/neu } \\
\text { expression }\end{array}$ & $\begin{array}{c}\text { Ki67 } \\
\text { expression }\end{array}$ & PCNA \\
\hline $\begin{array}{l}\text { Tauchi et al., } \\
1991 \text { (27) }\end{array}$ & & (-) Hsp70a & & & & & & & & \\
\hline $\begin{array}{l}\text { Jameel et al., } \\
1993 \text { (18) }\end{array}$ & (0) Hsp90 & & & (0) Hsp90 & $\begin{array}{l}\text { (+) Hsp90 } \\
\text { non ss }^{b}\end{array}$ & (0) Hsp90 & & & & \\
\hline $\begin{array}{l}\text { Shyamala et al., } \\
1993 \text { (22) }\end{array}$ & & & & & & $\begin{array}{c}\text { (+) Hsp90 } \\
\text { non ss }\end{array}$ & (0) Hsp90 & & & \\
\hline $\begin{array}{l}\text { Takahashi et al., } \\
1994 \text { (25) }\end{array}$ & (0) Hsp70 & & & & & (+) Hsp70 & & (0) Hsp70 & & \\
\hline $\begin{array}{l}\text { Takahashi et al., } \\
1995 \text { (26) }\end{array}$ & & & & & & (0) Hsp70 & & & & \\
\hline $\begin{array}{l}\text { Yano et al., } \\
1996 \text { (31) }\end{array}$ & & $\begin{array}{l}\text { (+) Hsp90a } \\
\text { (only for } \\
\text { mRNA ISH) }\end{array}$ & $\begin{array}{l}\text { (+) Hsp90b } \\
\text { (only for } \\
\text { mRNA ISH) }\end{array}$ & & & & & & & $\begin{array}{l}\text { (+) Hsp70 } \\
\text { nuclear } \\
(+) \text { Hsp90a } \\
\text { (0) Hsp90b } \\
\text { (only for } \\
\text { mRNA ISH) }\end{array}$ \\
\hline $\begin{array}{l}\text { Franzen et al., } \\
1997 \text { (17) }\end{array}$ & & (+) $\mathrm{Hsp} 90^{\mathrm{a}}$ & & & & & & & & \\
\hline $\begin{array}{l}\text { Lazaris et al., } \\
1997 \text { (20) }\end{array}$ & $\begin{array}{c}(0) \\
\text { Hsp70 }\end{array}$ & $\begin{array}{c}(+) \\
\text { Hsp70a }\end{array}$ & $\begin{array}{c}(+) \\
\text { Hsp70 }\end{array}$ & (0) Hsp70 & $\begin{array}{c}(+) \\
\text { Hsp70 }\end{array}$ & $\begin{array}{c}(0) \\
\text { Hsp70 }\end{array}$ & $\begin{array}{l}(+) \text { Hsp70 } \\
\text { non ss }\end{array}$ & & & (0) $\mathrm{Hsp} 0^{\mathrm{a}}$ \\
\hline $\begin{array}{l}\text { Vargas-Roig } \\
\text { et al., } 1997 \text { (30) }\end{array}$ & & & & & & & & & & $\begin{array}{l}\text { (0) Hsp70 for } \\
\text { entire tissue, } \\
(+) \text { Hsp70 } \\
\text { cytoplasmic, } \\
\text { cell by } \\
\text { cell analysis }\end{array}$ \\
\hline $\begin{array}{l}\text { Thanner } \text { et al., } \\
2003 \text { (28) }\end{array}$ & $\begin{array}{l}\text { (0) Hsp70 } \\
\text { nuclear } \\
(+) \text { Hsp70 } \\
\text { cyto- } \\
\text { plasmic } \\
\text { non ss }\end{array}$ & & $\begin{array}{l}\text { (+) Hsp70 } \\
\text { nuclear } \\
\text { (0) Hsp70 } \\
\text { cyto- } \\
\text { plasmic }\end{array}$ & $\begin{array}{l}\text { (+) Hsp70 } \\
\text { nuclear } \\
(0) \text { Hsp70 } \\
\text { cyto- } \\
\text { plasmic }\end{array}$ & & $\begin{array}{l}\text { (0) Hsp70 } \\
\text { nuclear } \\
\text { and } \\
\text { cyto- } \\
\text { plasmic }\end{array}$ & $\begin{array}{l}\text { (0) Hsp70 } \\
\text { nuclear, } \\
(+) \text { Hsp70 } \\
\text { cyto- } \\
\text { plasmic }\end{array}$ & & & \\
\hline $\begin{array}{l}\text { Torronteguy et al., } \\
2006 \text { (29) }\end{array}$ & & $\begin{array}{c}(+) \\
\text { Hsp70 }\end{array}$ & $\begin{array}{c}(0) \\
\text { Hsp70 }\end{array}$ & $\begin{array}{c}(0) \\
\text { Hsp70 }\end{array}$ & $\begin{array}{c}(0) \\
\text { Hsp70 }\end{array}$ & & & & & \\
\hline $\begin{array}{l}\text { Kalogeraki et al., } \\
2007 \text { (19) }\end{array}$ & & & $\begin{array}{c}(+) \\
\text { Hsp70 }\end{array}$ & & $\begin{array}{c}(+) \\
\text { Hsp70 }\end{array}$ & $\begin{array}{c}(+) \\
\text { Hsp70 }\end{array}$ & & & & $\begin{array}{c}(+) \\
\text { Hsp70 }\end{array}$ \\
\hline $\begin{array}{l}\text { Pick et al., } \\
2007 \text { (21) }\end{array}$ & $\begin{array}{c}(0) \\
\text { Hsp90 }\end{array}$ & & & $\begin{array}{c}(+) \\
\text { Hsp90 }\end{array}$ & $\begin{array}{c}(+) \\
\text { Hsp90 }\end{array}$ & $\begin{array}{c}(+) \\
\text { Hsp90 }\end{array}$ & $\begin{array}{c}(0) \\
\text { Hsp90 }\end{array}$ & $\begin{array}{c}(+) \\
\text { Hsp90 }\end{array}$ & & \\
\hline $\begin{array}{l}\text { Zagouri et al., } \\
2008 \text { (32) }\end{array}$ & & (-) Hsp90 & & & & (0) Hsp90 & & & & \\
\hline $\begin{array}{l}\text { Diehl et al., } \\
2009 \text { (2) }\end{array}$ & & $\begin{array}{l}\text { (0) Hsp90 } \\
\text { nuclear, } \\
\text { (+) Hsp90 } \\
\text { cytoplasmic }\end{array}$ & & & & $\begin{array}{l}\text { (-) Hsp90 } \\
\text { cytoplasmic } \\
\text { (0) Hsp90 } \\
\text { nuclear }\end{array}$ & (0) Hsp90 & & & \\
\hline $\begin{array}{l}\text { Batchelder } \text { et al., } \\
2009 \text { (14) }\end{array}$ & & $\begin{array}{c}\text { (-) Hsp70 } \\
\text { non ss }\end{array}$ & & & & & & & & \\
\hline $\begin{array}{l}\text { Zagouri et al., } 2010 \\
\text { (Ductal) (34) }\end{array}$ & & $\begin{array}{l}\text { (+) Hsp90 only } \\
\text { for IDC, }(-) \\
\text { Hsp90 in TNBC }\end{array}$ & $\begin{array}{l}(+) \text { Hsp90 } \\
\text { non ss }\end{array}$ & (+) Hsp90 & $\begin{array}{c}(0) \\
\text { Hsp90 }\end{array}$ & $\begin{array}{l}\text { (+) Hsp90, } \\
\text { but not in } \\
\text { multivariate }\end{array}$ & $\begin{array}{l}\text { (+) Hsp90, } \\
\text { but not in } \\
\text { multivariate }\end{array}$ & $\begin{array}{c}(+) \\
\text { Hsp90 }\end{array}$ & & \\
\hline Zagouri et al., & & $(-)$ & & & & (0) & (0) & $(0)$ & $(0)$ & \\
\hline 2010 (Lobular) (33) & & Hsp90 & & & & Hsp90 & Hsp90 & Hsp90 & Hsp90 & \\
\hline $\begin{array}{l}\text { Song et al., } 2010 \\
\text { (23) Dimas } \\
\text { (un-published } \\
\text { dataset) provided } \\
\text { by first author) }\end{array}$ & $\begin{array}{c}\text { (0) Hsp70 } \\
\text { and } \\
\text { Hsp90 }\end{array}$ & $\begin{array}{l}\text { (0) Hsp70, } \\
\text { (-) Hsp90 }\end{array}$ & $\begin{array}{c}\text { (0) Hsp90 } \\
\text { (0) Hsp70 } \\
\text { and } \\
\text { Hsp90 }\end{array}$ & $\begin{array}{l}\text { (0) Hsp90 } \\
\text { (0) Hsp70 } \\
\text { and } \\
\text { Hsp90 }\end{array}$ & $\begin{array}{c}\text { (0) Hsp90 } \\
\text { (0) Hsp70 } \\
\text { and } \\
\text { Hsp90 }\end{array}$ & $\begin{array}{c}\text { (0) Hsp90 } \\
\text { (0) Hsp70 } \\
\text { and } \\
\text { Hsp90 }\end{array}$ & $\begin{array}{l}\text { (0) Hsp70 } \\
\text { and } \\
\text { Hsp90 }\end{array}$ & $\begin{array}{l}\text { (0) Hsp90 } \\
\text { (0) Hsp70 } \\
\text { and } \\
\text { Hsp90 }\end{array}$ & $\begin{array}{c}\text { (0) Hsp90 } \\
\text { (0) Hsp70 } \\
\text { and } \\
\text { Hsp90 }\end{array}$ & \\
\hline
\end{tabular}

a: No statistical test performed; b: this association refers only to histologically confirmed nodal status; ss: statistically significant. 
a

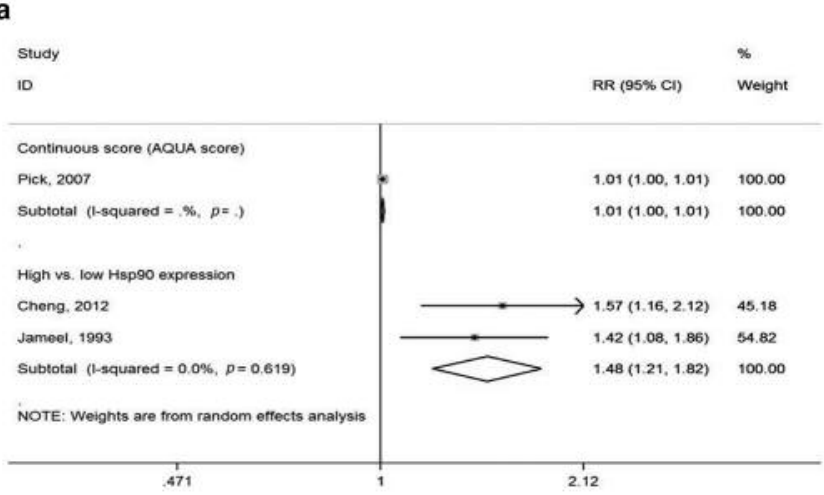

b

Stud

ID

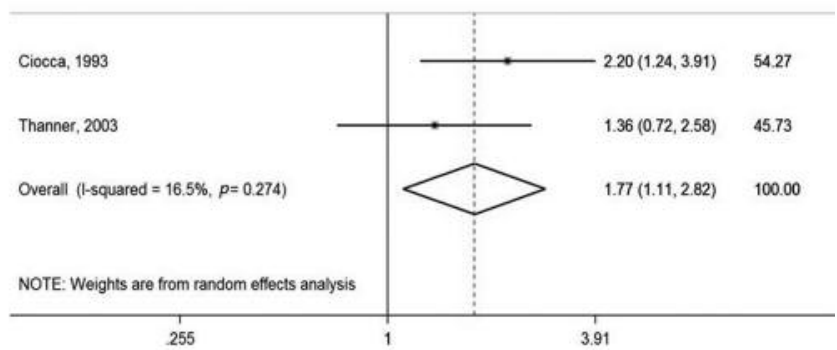

Figure 2. Forest plots presenting the association between a. Hsp90 expression and overall survival, and b. Hsp70 expression and disease-free survival.

a

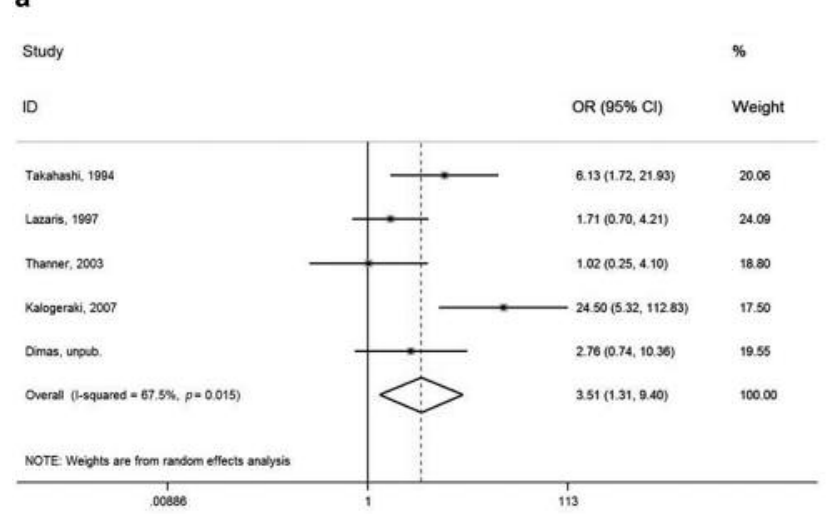

b

Study

ID

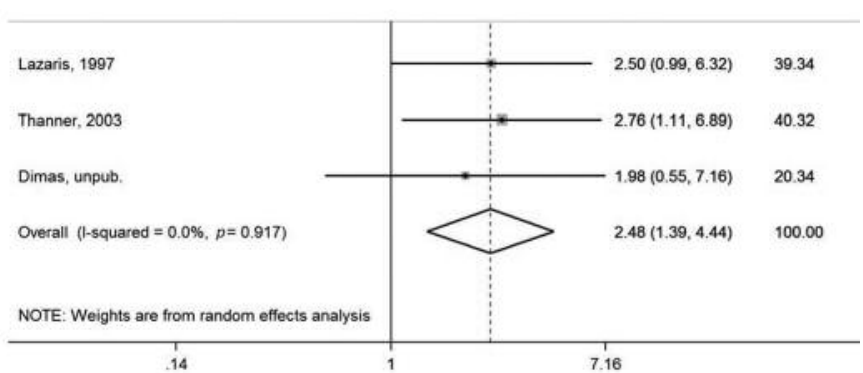

Figure 3. Forest plots presenting the association between $H$ sp70 expression and a. ER positivity, and b. PR positivity.

lost significance at the multivariate analysis) (34). There was another study that showed a similar association, but failed to reach statistical significance (22). However, Diehl et al. examining ductal carcinoma tissues, observed a significantly negative association with the cytoplasmic, but not nuclear, expression of Hsp90 (2). On the other hand, five studies, found no association $(9,18,23,32,33)$.

Similarly concerning progesterone receptor (PR) expression Pick et al., Shyamala et al., Zagouri et al., Diehl et al. and Dimas, unpub. found no association with Hsp90 (2, 9, 21, 22, 33). Zagouri et al. referring to ductal carcinomas indicated a positive correlation between the two, which however was not confirmed at a multivariate analysis (34).

With regard to HER2/neu, two studies found its positive correlation with Hsp90 expression $(21,34)$. On the contrary three more studies examining lobular, all types of breast carcinomas and ductal neoplasias of the breast, by Zagouri et al., Song et al. and Dimas, unpub. respectively, showed no correlation $(9,23,33)$.

Furthermore, three studies examined $\mathrm{Ki}-67$ and found no association with Hsp90 expression (9, 23, 33). Yano et al. examining Hsp90a and Hsp90b expression by means of mRNA ISH, showed a significant positive association with PCNALI for Hsp90a, but not for Hsp90b (31).

Hsp70 expression, subsequent development of metastases and survival. Three studies examined the impact of Hsp70 expression on OS. The studies by Ciocca et al. and Torronteguy et al. referring to all types of breast cancer showed no association $(16,29)$. Thanner et al., examining node negative carcinomas of the breast, showed a significantly worse survival of patients with high Hsp70 cytoplasmic expression, but no association could be established regarding nuclear Hsp70 expression (28). No synthesis was possible due to reporting reasons. 
However, in terms of DFS and RFS we found a statistically significant association between poorer DFS and high Hsp70 expression (pooled $\mathrm{RR}=1.77,95 \% \mathrm{CI}=1.71-2.82$ ), (Figure $2 \mathrm{~b}$ ). The alternative analysis based on nuclear Hsp70 expression (28) is presented in Supplemental File B (https://s3.eu-central1.amazonaws.com/dimas-et-al/ Supplemental+B.docx).

Torronteguy et al. showed a significant negative association between Hsp70 expression in invasive breast cancer and the development of distal metastases (29). However, Sun et al. found a significant positive association between high Hsp70 expression in triple negative breast cancer (TNBC) and the subsequent development of metastases. This association was not evident in $\operatorname{ER}(+) / \mathrm{PR}(+)$ breast cancer (24).

Hsp70 expression through the continuum of breast neoplasia. The relevant results are controversial; three studies encompassing all types of breast cancer presented a significantly higher Hsp70 expression (29), a lower Hsp70 expression (27) and a non-significant lower Hsp70 expression in cancerous tissues (14) respectively, compared to the noncancerous ones. A fourth study examining only ductal carcinomas not otherwise specified (DCNOS) produced a higher Hsp70 expression in the cancerous tissues (20). The unpublished cohort provided by the first author showed no difference in the expression of Hsp70 in cancerous vs. noncancerous tissues, as well as in invasive ductal carcinoma (IDC) $v s$. DCIS, and DCIS vs. normal breast tissue (9) (See also Supplemental File A (https://s3.eu-central1.amazonaws.com/ dimas-et-al/Supplemental+A.docx)).

Hsp70 expression in conjunction with clinicopathological variables. Takahashi et al., Lazaris et al., Thanner et al. and Dimas, unpub. addressed the possibility of an association between age and Hsp70 expression, which however was not proven significant $(9,20,25,28)$.

Regarding the correlation between Hsp70 expression and high histological grade three studies pertaining to DCNOS (20), node negative carcinomas of the breast (only as far as nuclear Hsp70 expression is concerned) (28) and IDC (19), showed significantly positive associations. Torronteguy et al. examining all types of breast cancer (29) and the Dimas, unpub. dataset examining only ductal neoplasia of the breast showed no association between histological grade and Hsp70 expression (9). The studies presenting data about odds ratio (OR) were synthesized and revealed no association between Hsp70 expression and histological grade (pooled $\mathrm{OR}=4.34,95 \% \mathrm{CI}=0.86-21.93$ ) (See also Supplemental File B (https:// s3.eu-central1.amazonaws.com/dimas-et-al/Supplemental+ B.docx)).

As far as tumor size is concerned there was a significant positive association between larger tumor size and Hsp70 expression in a study referring to the nuclear component of
Hsp70 in node negative breast carcinomas, but no such correlation was clear regarding its cytoplasmic expression (28). No clear difference in Hsp70 expression along with tumor size was noted in the study by Torronteguy et al. referring to all cancer types (29), the study by Lazaris et al. referring to DCNOS (20) and the unpublished cohort by Dimas, referring to ductal neoplasia of the breast (9). Confirming the individual studies, the meta-analysis yielded a null association (pooled $\mathrm{OR}=1.25,95 \% \mathrm{CI}=0.66-2.39$ ) (See also Supplemental File B (https://s3.eu-central-1.amazonaws.com/dimas-et-al/ Supplemental+B.docx )).

Regarding nodal metastases two studies showed a significant positive association with Hsp70 expression $(19,20)$, whereas two others $(9,29)$ showed no association. Overall the pooled association was null (pooled $\mathrm{OR}=3.53,95 \% \mathrm{CI}=0.71-17.60$ ) (See also Supplemental File B (https://s3.eu-central1.amazonaws.com/dimas-et-al/Supplemental+B.docx )).

As far as ER expression is concerned two studies, the first referring to ductal carcinomas (19) and the second examining all cancer types, by Takahashi et al. (25) showed a significant positive correlation with Hsp70 expression. However, a later study by Takahashi et al. examining the same specimens as the first, but this time paraffin-embedded and not fresh, found no association (26). No association was established in three other studies $(9,20,28)$. The quantitative synthesis yielded a significant positive association (pooled $\mathrm{OR}=3.51$, 95\%CI=1.31-9.40), (Figure 3a, See also Supplemental File B (https://s3.eu-central-1.amazonaws.com/dimas-etal/Supplemental+B.docx)).

Similarly, regarding PR expression, the quantitative synthesis highlighted a significant association between PR positivity and high Hsp70 expression (pooled $\mathrm{OR}=2.48$, $95 \% \mathrm{CI}=1.39-4.44) \quad(9,20,28) \quad$ (Figure 3b, See also Supplemental File B (https://s3.eu-central-1.amazonaws.com/ dimas-et-al/Supplemental+B.docx)).

The possible association between Hsp70 and HER2/neu expression was examined by two studies $(9,25)$, but no correlation was found. The only study examining Ki-67 was the unpublished one by Dimas, which found no association with Hsp70 (9).

Regarding PCNA labeling index two studies, (one on IDC (19) and another on all types (31)) presented a significant positive correlation. Another one referred to DCNOS and showed no correlation (20); lastly one referring to all breast cancer types showed a significant positive correlation between cytoplasmic Hsp70 expression and PCNALI when a cell-by-cell analysis was undertaken, whereas the analysis based on whole tissue samples showed no association (30).

Assessment of quality of studies. The assessment of quality of studies is presented in Supplemental File B (https://s3.eucentral-1.amazonaws.com/dimas-et-al/Supplemental+B.docx). 


\section{Discussion}

The prognostic significance of Hsps in breast cancer is better reflected on their impact on patients' survival. Increased Hsp90 expression was associated with 1.5-fold increased death rates; a finding of similar magnitude (1.5-fold) was noted regarding DFS, but remained only marginally significant, most probably due to the small number of eligible studies. In the instance of Hsp70 no unanimous conclusion could be reached regarding its association with OS. However, when looking into the effect of its expression (mainly cytoplasmic) on DFS, we found a 1.8-fold worse DFS in patients with high Hsp70 expression.

Hsp70 high expression was associated with 3.5-fold increased odds of ER positivity and accordingly with 2.5 fold increased odds of PR positivity. The fact that our results pointed to the same positive direction between Hsp70 expression, ER and PR respectively, is an indication of the internal consistency of our study findings, in view of the proven association between ER and PR positivity in breast cancer specimens (35). This knowledge adds to the assumption that an Hsp70 inhibitor could be an effective treatment for ER positive breast cancer, a welcome addition to the already established endocrine therapies for such tumors. Reporting reasons in the eligible studies did not allow us to quantitatively evaluate the association between Hsp90 expression and ER/PR positivity, highlighting a need for harmonized presentation of results in the various studies.

There was no conformity in the results pertaining to the remaining clinicopathological variables studied with regard to Hsp90/Hsp70 expression, including histological grade, tumor size, nodal metastasis or patients' age.

No clear differential pattern in the expression of Hsp70/Hsp90 along the breast neoplasia continuum was noted. More to the point, we documented that four studies examining Hsp90 showed a significantly higher expression in cancerous tissues compared to normal ones $(2,17,31,34)$. However, other studies showed a significantly lower expression in cancerous tissues (9, 32-34). Given these results, the question of Hsp90 levels along the breast neoplasia spectrum remains open, a fact that may have meaningful implications for the selectivity of these agents. Similarly, the results regarding Hsp70 were inconclusive; two studies showed a higher Hsp70 expression in cancerous tissues compared to the normal ones $(20,29)$, two demonstrated a lower Hsp70 expression in cancerous tissues $(14,27)$, whereas a fifth cohort found no difference (9).

Among the prerequisites for an effective new treatment is the selectivity of its agents, in order to reduce toxicities caused by damage to normal cells. The Hsp inhibitors were thought to play such a role, but their efficacy has yet to be proven in phase 3 clinical trials, according to a systematic review by our group (36). Presently the potency of those agents has mostly been suggested by studies on cancer cell lines and xenografts. However, the results of a phase 2 trial regarding ganetespib, an Hsp90 inhibitor, have implied a favorable outcome when it is used on selected patient subgroups (8). Also, Hsp70 inhibitors could play a role for a higher response to neoadjuvant aromatase inhibitor treatment (37).

Apart from the qualitative and quantitative synthesis, the present systematic review highlights limitations of the individual studies in terms of their quality ratings. Hsp70/Hsp90 expression was often assessed only by immunohistochemistry, a fact that may have limited the validity of the evaluation; approximately half of the studies adopted Western blotting, RT-PCR or immumohistochemical assessment by two expert pathologists, blind to the ratings of the other. Moreover, unadjusted effect estimates for clinicopathological variables were presented as a rule, a fact that may well imply the existence of confounding. In addition, in some longitudinal studies addressing survival, the follow-up was not long enough, being less than 5 years. Finally, markedly heterogeneous and often suboptimal reporting in numerous individual studies precluded their inclusion into the quantitative synthesis, a fact that may have limited the statistical power of this meta-analysis.

In conclusion, this systematic review and meta-analysis highlights the prognostic role of Hsp90 and Hsp70 expression in breast cancer, as evidenced by their associations with survival. Additional high-quality studies, with detailed reporting, seem desirable for further substantiation and validation of the present findings, as well as for a deeper understanding of the interplay between Hsp70/Hsp90 and clinicopathological parameters. The accumulation of such studies may well provide mechanistic, pathophysiological and epidemiological evidence complementing the findings of ongoing clinical trials on Hsp inhibitors.

\section{Conflicts of Interest}

The Authors declare no conflicts of interest.

\section{References}

1 Wang H, Tan MS, Lu RC, Yu JT and Tan L: Heat shock proteins at the crossroads between cancer and Alzheimer's disease. Biomed Res Int 2014: 239164, 2014.

2 Diehl MC, Idowu MO, Kimmelshue K, York TP, Elmore LW and Holt SE: Elevated expression of nuclear Hsp90 in invasive breast tumors. Cancer Biol Ther 8: 1952-1961, 2009.

3 Jhaveri K and Modi S: HSP90 inhibitors for cancer therapy and overcoming drug resistance. Adv Pharmacol 65: 471-517, 2012.

4 Neckers L and Workman P: Hsp90 molecular chaperone inhibitors: are we there yet? Clin Cancer Res 18: 64-76, 2012.

5 Isambert N, Delord JP, Soria JC, Hollebecque A, Gomez-Roca C, Purcea D, Rouits E, Belli R and Fumoleau P: Debio0932, a second-generation oral heat shock protein (HSP) inhibitor, in patients with advanced cancer-results of a first-in-man dose- 
escalation study with a fixed-dose extension phase. Ann Oncol 26: 1005-1011, 2015.

6 Wang X, Chen M, Zhou J and Zhang X: HSP27, 70 and 90, antiapoptotic proteins, in clinical cancer therapy (Review). Int $\mathrm{J}$ Oncol 45: 18-30, 2014.

7 Gaykema SB, Schroder CP, Vitfell-Rasmussen J, Chua S, Oude Munnink TH, Brouwers AH, Bongaerts AH, Akimov $M$, Fernandez-Ibarra C, Lub-de Hooge MN, de Vries EG, Swanton $\mathrm{C}$ and Banerji U: 89Zr-trastuzumab and 89Zr-bevacizumab PET to evaluate the effect of the HSP90 inhibitor NVP-AUY922 in metastatic breast cancer patients. Clin Cancer Res 20: 39453954, 2014.

8 Jhaveri K, Chandarlapaty S, Lake D, Gilewski T, Robson M, Goldfarb S, Drullinsky P, Sugarman S, Wasserheit-Leiblich C, Fasano J, Moynahan ME, D'Andrea G, Lim K, Reddington L, Haque S, Patil S, Bauman L, Vukovic V, El-Hariry I, Hudis C and Modi S: A phase II open-label study of ganetespib, a novel heat shock protein 90 inhibitor for patients with metastatic breast cancer. Clin Breast Cancer 14: 154-160, 2014.

9 Dimas D: Heat shock protein 70 and 90 immunohistochemical expression in ductal neoplasia of the breast. National and Kapodistrian University of Athens, 2015.

10 Higgins JPT and Green S: Cochrane Handbook for Systematic Reviews of Interventions Version 5.1.0 [updated March 2011]. In: The Cochrane Collaboration, 2011.

11 Gialamas SP, Sergentanis TN, Antonopoulos CN, Dessypris N, Chrousos GP and Petridou ET: Circulating leptin levels and risk of colorectal cancer and adenoma: a case-control study and meta-analysis. Cancer Causes Control 24: 2129-2141, 2013.

12 Petridou ET, Sergentanis TN, Perlepe C, Papathoma P, Tsilimidos G, Kontogeorgi E, Kourti M, Baka M, Moschovi M, Polychronopoulou S, Sidi V, Hatzipantelis E, Stiakaki E, Iliadou AN, La Vecchia C, Skalkidou A and Adami HO: Socioeconomic disparities in survival from childhood leukemia in the United States and globally: a meta-analysis. Ann Oncol 26: 589-597, 2015.

13 Psaltopoulou T, Sergentanis TN, Panagiotakos DB, Sergentanis IN, Kosti $\mathrm{R}$ and Scarmeas N: Mediterranean diet, stroke, cognitive impairment, and depression: A meta-analysis. Ann Neurol 74: 580-591, 2013

14 Batchelder AJ, Gordon-Weeks AN and Walker RA: Altered expression of anti-apoptotic proteins in non-involved tissue from cancer-containing breasts. Breast Cancer Res Treat 114: 63-69, 2009.

15 Cheng Q, Chang JT, Geradts J, Neckers LM, Haystead T, Spector NL and Lyerly HK: Amplification and high-level expression of heat shock protein 90 marks aggressive phenotypes of human epidermal growth factor receptor 2 negative breast cancer. Breast Cancer Res 14: R62, 2012.

16 Ciocca DR, Clark GM, Tandon AK, Fuqua SA, Welch WJ and McGuire WL: Heat shock protein hsp70 in patients with axillary lymph node-negative breast cancer: prognostic implications. J Natl Cancer Inst 85: 570-574, 1993.

17 Franzen B, Linder S, Alaiya AA, Eriksson E, Fujioka K, Bergman AC, Jornvall $\mathrm{H}$ and Auer G: Analysis of polypeptide expression in benign and malignant human breast lesions. Electrophoresis 18: 582-587, 1997.

18 Jameel A, Law M, Coombes R and Luqmani Y: Significance of heat-shock protein-90 as a prognostic indicator in breast-cancer. Int J Oncol 2: 1075-1080, 1993.
19 Kalogeraki A, Giannikaki E, Tzardi M, Kafousi M, Ieromonachou P, Dariviannaki K, Askoxylakis J, Tsiftsis D, Stathopoulos E and Zoras O: Correlation of heat shock protein (HSP70) expression with cell proliferation (MIB1), estrogen receptors (ER) and clinicopathological variables in invasive ductal breast carcinomas. J Exp Clin Cancer Res 26: 367-368, 2007.

20 Lazaris A, Chatzigianni EB, Panoussopoulos D, Tzimas GN, Davaris PS and Golematis B: Proliferating cell nuclear antigen and heat shock protein 70 immunolocalization in invasive ductal breast cancer not otherwise specified. Breast Cancer Res Treat 43: 43-51, 1997.

21 Pick E, Kluger Y, Giltnane JM, Moeder C, Camp RL, Rimm DL and Kluger HM: High HSP90 expression is associated with decreased survival in breast cancer. Cancer Res 67: 2932-2937, 2007.

22 Shyamala G, Schweitzer M and Ullrich SJ: Relationship between 90-kilodalton heat shock protein, estrogen receptor, and progesterone receptor in human mammary tumors. Breast Cancer Res Treat 26: 95-100, 1993.

23 Song CH, Park SY, Eom KY, Kim JH, Kim SW, Kim JS and Kim IA: Potential prognostic value of heat-shock protein 90 in the presence of phosphatidylinositol-3-kinase overexpression or loss of PTEN, in invasive breast cancers. Breast Cancer Res 12: R20, 2010.

24 Sun B, Zhang S, Zhang D, Li Y, Zhao X, Luo Y and Guo Y: Identification of metastasis-related proteins and their clinical relevance to triple-negative human breast cancer. Clin Cancer Res 14: 7050-7059, 2008.

25 Takahashi S, Mikami T, Watanabe Y, Okazaki M, Okazaki Y, Okazaki A, Sato T, Asaishi K, Hirata K, Narimatsu E et al: Correlation of heat shock protein 70 expression with estrogen receptor levels in invasive human breast cancer. Am J Clin Pathol 101: 519-525, 1994.

26 Takahashi S, Narimatsu E, Asanuma H, Okazaki M, Okazaki A, Hirata K, Mori $\mathrm{M}$, Chiba $\mathrm{T}$, Sato $\mathrm{N}$ and Kikuchi $\mathrm{K}$ : Immunohistochemical detection of estrogen receptor in invasive human breast cancer: correlation with heat shock proteins, pS2 and oncogene products. Oncology 52: 371-375, 1995.

27 Tauchi K, Tsutsumi Y, Hori S, Yoshimura S, Osamura RY and Watanabe K: Expression of heat shock protein 70 and c-myc protein in human breast cancer: an immunohistochemical study. Jpn J Clin Oncol 21: 256-263, 1991

28 Thanner F, Sutterlin MW, Kapp M, Rieger L, Kristen P, Dietl J, Gassel AM and Muller T: Heat-shock protein 70 as a prognostic marker in node-negative breast cancer. Anticancer Res 23: 10571062, 2003.

29 Torronteguy C, Frasson A, Zerwes F, Winnikov E, da Silva VD, Menoret $\mathrm{A}$ and Bonorino $\mathrm{C}$ : Inducible heat shock protein 70 expression as a potential predictive marker of metastasis in breast tumors. Cell Stress Chaperones 11: 34-43, 2006.

30 Vargas-Roig LM, Fanelli MA, Lopez LA, Gago FE, Tello O, Aznar JC and Ciocca DR: Heat shock proteins and cell proliferation in human breast cancer biopsy samples. Cancer Detect Prev 21: 441-451, 1997.

31 Yano M, Naito Z, Tanaka S and Asano G: Expression and roles of heat shock proteins in human breast cancer. Jpn J Cancer Res 87: 908-915, 1996.

32 Zagouri F, Nonni A, Sergentanis TN, Papadimitriou CA, Michalopoulos NV, Lazaris AC, Patsouris E and Zografos GC: Heat shock protein90 in lobular neoplasia of the breast. BMC Cancer 8: 312, 2008. 
33 Zagouri F, Sergentanis T, Nonni A, Papadimitriou C, Pazaiti A, Michalopoulos NV, Safioleas P, Lazaris A, Theodoropoulos G, Patsouris E and Zografos G: Decreased Hsp90 expression in infiltrative lobular carcinoma: an immunohistochemical study. BMC Cancer 10: 409, 2010.

34 Zagouri F, Sergentanis TN, Nonni A, Papadimitriou CA, Michalopoulos NV, Domeyer P, Theodoropoulos G, Lazaris A, Patsouris E, Zogafos E, Pazaiti A and Zografos GC: Hsp90 in the continuum of breast ductal carcinogenesis: Evaluation in precursors, preinvasive and ductal carcinoma lesions. BMC Cancer 10: 353, 2010.

35 Yip $\mathrm{CH}$ and Rhodes A: Estrogen and progesterone receptors in breast cancer. Future Oncol 10: 2293-2301, 2014.
36 Zagouri F, Sergentanis TN, Chrysikos D, Papadimitriou CA, Dimopoulos MA and Psaltopoulou T: Hsp90 inhibitors in breast cancer: a systematic review. Breast 22: 569-578, 2013.

37 Yiu CC, Chanplakorn N, Chan MS, Loo WT, Chow LW, Toi M and Sasano H: Down-regulation of heat-shock protein 70 (HSP70) correlated with responsiveness to neoadjuvant aromatase inhibitor therapy in breast cancer patients. Anticancer Res 30: 3465-3472, 2010.

Received October 6, 2017

Revised December 16, 2017

Accepted December 19, 2017 